'Departamento de Nutrición, Clínica Las Condes, Santiago, Chile.

${ }^{2}$ Clínica de Lípidos, Departamento Medicina Interna, Hospital Universitario Fundación Jiménez Díaz, Madrid, España.

Ubicación actual: Departamento de Nutrición, Clínica Las Condes. Santiago de Chile.

Fuente de apoyo financiero: Ninguna.

Recibido el 27 de septiembre de 2013, aceptado el 22 de enero de 2014.

Correspondencia a: Dra. Ada Cuevas Marín Departamento de Nutrición Clínica Las Condes. Lo Fontecilla 441, Santiago, Chile. Fono: 562-26108561 Fax: 562-26108565 acuevas@clc.cl

\section{Nuevos fármacos para el tratamiento de la hipercolesterolemia}

\author{
ADA CUEVAS ${ }^{1}$, MARÍA MAGDALENA FARÍAS ${ }^{1}$, RODRIGO ALONSO $^{2}$
}

\section{New lipid lowering agents}

Statins are the preferred treatment for hypercholesterolemia and several studies have demonstrated their long-term safety and efficacy in reducing cardiovascular morbidity and mortality. However, in some cases of severe hypercholesterolemia such as homozygous and heterozygous familial hypercholesterolemia or statin intolerant patients, statins can be less efficient. In recent years, new lipid-lowering agents with novel mechanisms of action have been developed to reduce LDL-cholesterol in patients with severe hypercholesterolemia, associated or not to conventional lipid-lowering therapy. These therapies include microsomal transfer protein inhibitor (Lomitapide), antisense oligonucleotide to Apo B100 (Mipomersen) and monoclonal antibodies against Proprotein convertase subtilisin/kexin type 9 (PCSK9). Different studies have shown the great effectiveness of these new therapies. Short-term studies confirmed their adequate security profile, especially in patients with homozygous familiar hypercholesterolemia or severe hypercholesterolemia. Some of these agents have been also tested in statin-intolerant patients. However, long-term studies are needed to evaluate their safety, effectiveness and impact on cardiovascular risk reduction.

(Rev Med Chile 2014; 142: 880-888)

Key words: Anticholesteremic agents; Hypercholesterolemia, familial combined; Lipido-lowering therapy; Lomitapide; Mipomersen.

\section{L} a hipercolesterolemia familiar (HF) es el trastorno monogénico más frecuente asociado a enfermedad coronaria prematura (ECP). Se produce por mutaciones en el gen del receptor de las lipoproteínas de baja densidad (RLDL) y menos frecuentemente por mutaciones en el gen de la apolipoproteína B (ApoB) y de la proproteína convertasa subtilisina/kexina tipo 9 (PCSK9) ${ }^{1-3}$. Se hereda de forma autosómica dominante y tiene una penetrancia cercana a $100 \%{ }^{1}$. Existen dos formas de HF, la heterocigota $(\mathrm{HFH})$, cuando se hereda sólo un alelo mutado de uno de los progenitores, y la homocigota (HFHo), cuando se hereda de ambos progenitores la misma mutación. La HFH compuesta se produce cuando se hereda una mutación distinta en el mismo gen de cada progenitor y tiene una expresión clínica similar a la HFHo. La prevalencia de la HFH en la población general es de 1 caso en
300-500 personas, por lo que en Chile se estima que pueden existir de 30.000 a 40.000 sujetos con este trastorno. En cambio, la HFHo se considera una enfermedad rara ya que su prevalencia es de 1 caso por millón de habitantes.

La HF se caracteriza por presentar niveles elevados de colesterol total y colesterol transportado por las lipoproteínas de baja densidad (C-LDL), ECP y xantomas tendinosos, siendo la expresión clínica mucho más severa y de aparición más temprana en la $\mathrm{HFHo}^{4}$.

Los pacientes con HFHo presentan una gran carga de enfermedad ateroesclerótica por la exposición a niveles elevados de colesterol desde el nacimiento. Por este motivo, requieren tratamiento farmacológico intenso desde edades muy tempranas, incluida la LDL-aféresis ${ }^{4,5}$. La mayoría de los pacientes homocigotos no logran reducir de manera significativa sus niveles de C-LDL con el 
tratamiento farmacológico convencional debido a la ausencia o falta de actividad de los RLDL, y persisten con un elevado riesgo de desarrollar $\mathrm{ECP}^{6}$.

En los últimos años se han desarrollado nuevos fármacos reductores del C-LDL con mecanismos de acción distintos a los ya conocidos. Estos corresponden a los inhibidores de la proteína de transferencia de triglicéridos microsomales (MTP) como lomitapide, los fármacos antisentido ApoB-100 como mipomersen y los anticuerpos monoclonales anti-PCSK9. Recientemente, la US Food and Drug Administration (FDA) en Estados Unidos de Norteamérica ha autorizado la indicación de lomitapide y mipomersen para el tratamiento de la $\mathrm{HFHo}^{7}$, y la Agencia Europea del Medicamento (EMA) ha autorizado lomitapide ${ }^{8}$. Los anti-PCSK9 se encuentran en estudios en fase III, dirigidos entre otros a la HFH y a la HFHo.

El objetivo de esta revisión es conocer los mecanismos de acción, eficacia y seguridad de estos nuevos fármacos hipolipemiantes y su papel en el futuro del tratamiento de la hipercolesterolemia severa.

\section{Lomitapide (AEGR-733, Juxtapid ${ }^{\circledR}$ en Estados Unidos de Norteamérica y Lojuxta ${ }^{\circledR}$ en Europa)}

Es una molécula desarrollada para ser administrada vía oral que inhibe la proteína de transferencia de triglicéridos microsomales (MTP) lo que resulta en una reducción del C-LDL y de los triglicéridos plasmáticos ${ }^{9}$. En Estados Unidos de Norteamérica y en Europa ha sido autorizado para el tratamiento de la HFHo en adultos mayores de 18 años como terapia coadyudante a una dieta baja en grasas y al tratamiento farmacológico hipolipemiante convencional junto a LDL-aféresis si está disponible ${ }^{7,8}$.

La MTP es una proteína esencial para el ensamblaje y secreción de las lipoproteínas de muy baja densidad (VLDL) de origen hepático y de los quilomicrones de origen intestinal ${ }^{10}$. La importancia de esta proteína como objetivo de tratamiento surgió al estudiar los casos con abetalipoproteinemia, una enfermedad rara producida por mutaciones en esta enzima, que demostró que estos pacientes tienen niveles muy bajos de colesterol y triglicéridos ${ }^{11}$.

La eficacia de lomitapide en monoterapia se evaluó en un estudio en fase II "Prueba de con- cepto" en 6 pacientes con HFHo > 18 años, con titulación forzada de dosis durante 16 semanas $(\text { Tabla } 1)^{12}$. Los pacientes siguieron una dieta muy estricta $(<10 \%$ del aporte energético como grasas), junto a suplementación de vitaminas y ácidos grasos esenciales. La reducción máxima en C-LDL y triglicéridos fue de $51 \%$ y $65 \%$, respectivamente. Los efectos adversos más frecuentes ocurrieron a nivel gastrointestinal asociado a un mayor consumo de grasas en la dieta. También se observó elevación de las transaminasas y aumento en el contenido de grasa hepática que se normalizaron a las 4 semanas de haber finalizado el tratamiento ${ }^{12}$.

La eficacia y seguridad de lomitapide como terapia coayudante a otros tratamientos hipolipemiantes incluyendo la LDL-aféresis se evaluó en un estudio fase III, abierto, no aleatorizado que incluyó a 29 pacientes adultos con $\mathrm{HFHo}^{13}$. El estudio tuvo una primera fase de eficacia de 26 semanas (objetivo primario) y otra de seguridad de la semana 26 a la 78 . En la fase de eficacia, el tratamiento farmacológico concomitante y la LDL-aféresis no se modificaron, pero si se hicieron modificaciones en la fase de seguridad. Los pacientes recibieron el fármaco en dosis de $5 \mathrm{mg}$ con escalada progresiva hasta $60 \mathrm{mg} /$ día o hasta la dosis máxima tolerada. De los 29 sujetos, 23 completaron las dos fases. La reducción media en C-LDL en la semana 26 fue de $40 \%$ en los 29 sujetos (intención de tratamiento) y de $50 \%$ en los que completaron el estudio, manteniéndose en torno a $40 \%$ al final de la fase de seguridad (semana 78). También se observó una reducción significativa en ApoB, triglicéridos y lipoproteína(a) [Lp(a)] al finalizar la fase de eficacia, siendo menor el efecto en la semana 78, probablemente por los cambios producidos en la terapia concomitante durante la fase de seguridad.

Los efectos más frecuentes ocurrieron a nivel gastrointestinal (diarrea, náuseas y dispepsia, entre otros) y hepático. Se notificaron reacciones adversas gastrointestinales de intensidad grave en $6(21 \%)$ de 29 pacientes y 4 pacientes discontinuaron el fármaco por efectos secundarios gastrointestinales durante la fase de eficacia y ninguno durante la fase de seguridad. El aumento de las transaminasas $\geq 3$ veces el valor normal en al menos una ocasión se evidenció en 10 sujetos y en ninguno fue motivo para discontinuar el fármaco. Estos efectos disminuyeron con la reducción de dosis o la discontinuación del lomitapide en un 
Tabla 1. Estudios con Mipomersen y Lomitapide

\begin{tabular}{|c|c|c|c|c|c|}
\hline Estudio & Fármaco/dosis & Población (n) & Duración & $\begin{array}{l}\text { Reducción } \\
\text { C-LDL (\%) }\end{array}$ & $\begin{array}{l}\text { Reducción } \\
\operatorname{Lp}(\mathrm{a})(\%)\end{array}$ \\
\hline $\begin{array}{l}\text { Cuchel M } \\
\text { et } \mathrm{al}^{12}\end{array}$ & $\begin{array}{l}\text { Lomitapide } \\
0,03 \text { a } 1,0 \mathrm{mg} / \mathrm{kg} / \\
\text { día c/4 semanas, } \\
\text { vo }\end{array}$ & $\begin{array}{l}6 \text { HFHo } 18-40 \text { años } \\
\text { (discontinuación previa de } \\
\text { tratamientos hipolipemiantes, } \\
\text { incluida LDL-aféresis) }\end{array}$ & 20 semanas & $3,7 \%-50,9 \%$ & No disponible \\
\hline $\begin{array}{l}\text { Cuchel M } \\
\text { et } \mathrm{al}^{13}\end{array}$ & $\begin{array}{l}\text { Lomitapide } \\
\text { Escalada dosis } \\
\text { de } 5 \text { a } 60 \mathrm{mg} / \mathrm{día} \\
\text { c/4 semanas }\end{array}$ & $\begin{array}{l}29 \text { HFHo } \geq 18 \text { años con } \\
\text { tratamiento hipolipemiante o } \\
\text { LDL-aféresis }\end{array}$ & $\begin{array}{l}26 \text { semanas } \\
\text { (fase eficacia) } \\
78 \text { semanas } \\
\text { (fase seguridad) }\end{array}$ & $\begin{array}{c}40,0 \% \text { (intención } \\
\text { tratamiento) } \\
38,0 \%\end{array}$ & $\begin{array}{l}-15,0 \% \\
-1,0 \%\end{array}$ \\
\hline $\begin{array}{l}\text { Akdim } F \\
\text { et } \mathrm{al}^{20}\end{array}$ & $\begin{array}{l}\text { Mipomersen } \\
50 \text { a } 300 \mathrm{mg} \\
\text { sc/ semana }\end{array}$ & $\begin{array}{l}44 \mathrm{HFH} \text { con tratamiento } \\
\text { hipolipemiante }\end{array}$ & 6 semanas & $13,0 \%-34,0 \%$ & $3,0 \%-24,0 \%$ \\
\hline $\begin{array}{l}\text { Akdim F } \\
\text { et } \mathrm{al}^{21}\end{array}$ & $\begin{array}{l}\text { Mipomersen } \\
200 \mathrm{mg} \mathrm{sc} / \text { semana } \\
\text { x } 12 \mathrm{sem}\end{array}$ & $\begin{array}{l}74 \mathrm{HCol}, \mathrm{C}-\mathrm{LDL} 100-200 \mathrm{mg} / \\
\mathrm{dL} \text { con tratamiento estable } \\
\text { con estatinas }\end{array}$ & $\begin{array}{l}30 \text { días desde } \\
\text { la última dosis }\end{array}$ & $35,7 \%$ & No disponible \\
\hline $\begin{array}{l}\text { Akdim F } \\
\text { et } a^{22}\end{array}$ & $\begin{array}{l}\text { Mipomersen } \\
50 \text { a } 400 \mathrm{mg} \\
\text { sc/semana }\end{array}$ & $\begin{array}{l}50 \mathrm{HCol}, \mathrm{C}-\mathrm{LDL} 110-226 \mathrm{mg} / \\
\mathrm{dL} \text { sin tratamiento hipolipe- } \\
\text { miante }\end{array}$ & 13 semanas & $7,0 \%-71,0 \%$ & $49,0 \%$ \\
\hline $\begin{array}{l}\text { Raal F } \\
\text { et al } 23\end{array}$ & $\begin{array}{l}\text { Mipomersen } 200 \\
\text { mg sc c/semana } \\
\text { vs placebo }\end{array}$ & $\begin{array}{l}51 \mathrm{HFHo} \geq 12 \text { años con } \\
\text { máximo tratamiento hipoli- } \\
\text { pemiante }\end{array}$ & 26 semanas & $24,7 \%$ vs $3,3 \%$ & $31,8 \%$ vs $7,0 \%$ \\
\hline $\begin{array}{l}\text { Stein EA } \\
\text { et } \mathrm{al}^{24}\end{array}$ & $\begin{array}{l}\text { Mipomersen } 200 \\
\text { mg sc c/semana } \\
\text { vs placebo }\end{array}$ & $\begin{array}{l}124 \mathrm{HFH} \text { con EC,C-LDL } \\
\geq 100 \mathrm{mg} / \mathrm{dL}\end{array}$ & 26 semanas & $28,0 \%$ vs $+5,2 \%$ & $21,0 \%$ vs $0 \%$ \\
\hline $\begin{array}{l}\text { McGowan M } \\
\text { et all } 25\end{array}$ & $\begin{array}{l}\text { Mipomersen } 200 \\
\text { mg sc c/semana } \\
\text { vs placebo }\end{array}$ & $\begin{array}{l}58 \text { hipercolesterolemia severa } \\
\text { (C-LDL } \geq 300 \mathrm{mg} / \mathrm{dL} \text { o } \\
\geq 200 \mathrm{mg} / \mathrm{dL} \text { con } \mathrm{ECV} \text {, } \\
\text { máximo tratamiento hipoli- } \\
\text { pemiante }\end{array}$ & 26 semanas & $36,0 \%$ vs $+12,5 \%$ & $32,7 \%$ vs $1,5 \%$ \\
\hline $\begin{array}{l}\text { Visseres M } \\
\text { et } \mathrm{al}^{26}\end{array}$ & $\begin{array}{l}\text { Mipomersen } 200 \\
\text { mg sc c/semana } \\
\text { vs placebo }\end{array}$ & $\begin{array}{l}34 \mathrm{Hcol} \text { intolerantes a esta- } \\
\text { tinas y alto riesgo cardiovas- } \\
\text { cular }\end{array}$ & 26 semanas & $47,3 \%$ vs $2,0 \%$ & $27,1 \%$ vs $0 \%$ \\
\hline $\begin{array}{l}\text { Thomas GS } \\
\text { et } \mathrm{a}^{27}\end{array}$ & $\begin{array}{l}\text { Mipomersen } 200 \\
\text { mg sc c/semana } \\
\text { vs placebo }\end{array}$ & $\begin{array}{l}158 \text { sujetos con enfermedad } \\
\text { coronaria o alto } \mathrm{RCV} \text { (incluye } \\
\text { DM2) y LDL } \geq 100 \mathrm{mg} / \mathrm{dL} \text { en } \\
\text { tratamiento hipolipemiante }\end{array}$ & 26 semanas & $36,9 \%$ vs $4,5 \%$ & $25,6 \%$ vs $0 \%$ \\
\hline
\end{tabular}

$\mathrm{HFH}$, hipercolesterolemia familiar heterocigota; HFHo, hipercolesterolemia familiar homocigota; Hcol, hipercolesterolemia; RCV, riesgo cardiovascular; DM2, Diabetes mellitus tipo 2; ECV, enfermedad cardiovascular; sc, subcutáneo; v.o, vía oral; vs, versus.

período de 4 semanas ${ }^{13}$. Además, se evidenció mediante resonancia magnética nuclear, un aumento de $8,6 \%$ en el contenido en grasa del hígado al final de la fase de eficacia que se mantuvo constante durante el estudio. Este efecto se explica por el mecanismo de acción del fármaco y parece ser reversible al discontinuar el tratamiento ${ }^{13}$.

Lomitapide se metaboliza principalmente por el citocromo P450 3A4 (CYP3A4), por tanto, el uso concomitante de inhibidores moderadospotentes de CYP3A4 como antifúngicos azólicos, antibióticos macrólidos, inhibidores de la proteasa y bloqueadores de canales de calcio, entre otros está contraindicado ${ }^{14}$. Lomitapide puede aumentar las concentraciones plasmáticas de las estatinas, lo que tiene importancia práctica ya que estas son 
de primera elección en los pacientes con HF. En personas sanas, $10 \mathrm{mg}$ de lomitapide aumenta de forma clínicamente significativa los niveles de simvastatina, pero no los de atorvastatina y rosuvastatina ${ }^{9}$.

Desde un punto de vista práctico, se sugiere comenzar con la dosis de $5 \mathrm{mg}$ de lomitapide una vez al día ( 2 h después de la cena) y titular la dosis cada 4 semanas según tolerancia (máximo $60 \mathrm{mg}$ / día). De acuerdo a la ficha técnica del fármaco aprobada por las agencias regulatorias (EMA y FDA), el paciente que recibe este fármaco debe hacer una dieta estricta con menos de $20 \%$ de aporte calórico en grasas y con suplementación de vitamina $\mathrm{E}$ y ácidos grasos esenciales. No se debe utilizar simvastatina en dosis $>40 \mathrm{mg}$ diarios. Deben vigilarse periódicamente las transaminasas y se recomienda hacer un tamizado de esteatohepatitis y fibrosis hepática si se evidencia aumento del contenido graso en el hígado.

\section{Mipomersen (Genzyme/ISIS 301012, Kinamro ${ }^{\circledR}$ en Estados Unidos de Norteamérica)}

Mipomersen es un oligonucleótido antisentido de segunda generación diseñado para unirse a una secuencia específica de bases del ARN mensajero (ARNm) de la ApoB-10015. Como resultado se produce una inhibición en la síntesis hepática de esta apolipoproteína que es crucial en la producción de las LDL y otras lipoproteínas aterogéni$\mathrm{cas}^{16}$. Se distribuye ampliamente por los distintos tejidos del organismo, con excepción del músculo esquelético, alcanzando su mayor concentración en hígado y riñón. Tiene una vida media de 23 a 46 días dependiendo de la dosis ${ }^{17}$, se metaboliza por endo y exonucleasas y su eliminación es por vía renal ${ }^{18}$. No se metaboliza y tampoco inhibe las isoenzimas del citocromo P450, por lo tanto, no se esperan interacciones significativas con otros fármacos como estatinas o ezetimiba ${ }^{19}$.

El medicamento ha sido aprobado recientemente por la FDA como droga huérfana para el tratamiento de la HFHo, en combinación con otros agentes hipolipemiantes y cambios de estilo de vida ${ }^{7}$.

Su efecto en la reducción de C-LDL y ApoB100 depende de la dosis y del tiempo de exposición al fármaco. La dosis de administración es de 200 mg una vez por semana y por vía subcutánea (sc).
Los estudios en fase II, aleatorizados y dobleciego, realizados en pacientes con $\mathrm{HFH}^{20}$, y en pacientes con hipercolesterolemia con o sin tratamiento con estatinas ${ }^{21,22}$ han permitido evaluar la seguridad, eficacia y dosificación de mipomersen (Tabla 1). En todos los estudios se observó una reducción del C-LDL que es dosis-dependiente, alcanzando $34 \%$ en la HFH (20), 37,5\% en los hipercolesterolemicos en tratamiento con estatinas ${ }^{21}, y$ $71 \%$ cuando se administró en monoterapia ${ }^{22}$. En estos estudios, se observó también una reducción significativa de los niveles de Lp(a), siendo de $17 \%$ en los pacientes con HFH en la dosis de $200 \mathrm{mg}$ y de $42 \%$ en los pacientes con hipercolesterolemia sin tratamiento con estatinas. Los principales efectos adversos asociados al fármaco fueron las reacciones en el lugar de la aplicación, síntomas similares a la gripe y la elevación discreta de las transaminasas. A partir de estos y otros estudios en fase 2, se seleccionó la dosis de $200 \mathrm{mg}$ semanales para las evaluaciones posteriores en estudios en fase 3.

La molécula ha sido evaluada en 5 estudios fase III (Tabla 1) incluyendo 425 pacientes con $\mathrm{HFH}$ y $\mathrm{HFHo}^{23,24}$, hipercolesterolemia severa ${ }^{25}$, pacientes intolerantes al uso de estatinas con riesgo cardiovascular muy elevado ${ }^{26}$, y pacientes con alto riesgo cardiovascular y/o enfermedad coronaria en tratamiento hipolipemiante ${ }^{27}$. En estos 5 estudios, los pacientes fueron aleatorizados para recibir 200 mg de mipomersen una vez por semana o placebo vía sc durante 26 semanas en combinación con el tratamiento hipolipemiante y el objetivo primario fue la reducción de C-LDL a las 2 semanas de la última dosis del fármaco. En todos los estudios se obtuvo una reducción significativa que varió de $24,7 \%$ en los pacientes homocigotos ${ }^{23}$ hasta $47,3 \%$ obtenido en los pacientes intolerantes a estatinas ${ }^{26}$. Además, se observó una reducción significativa en las concentraciones plasmáticas de Lp(a) de aproximadamente $25 \%$. El mecanismo de acción por el cual mipomersen reduce los niveles de lipoproteina(a) no está aun establecido. En general, el máximo efecto reductor de C-LDL se obtiene a las 5 semanas de iniciado el tratamiento, manteniéndose estable hasta dos semanas después de suspendido.

Los análisis de seguridad han demostrado que mipomersen es en general bien tolerado. Los efectos adversos más frecuentes son las reacciones en el sitio de inyección (80-100\%) tales como cambio 
de coloración en la piel, enrojecimiento y prurito entre otros, que aunque leves son una de las causas frecuentes de discontinuación del tratamiento. Los síntomas similares a la gripe ocurren en 30\% a $50 \%$ de los pacientes y la elevación de las transaminasas $\geq 3$ veces el valor normal varía en los distintos estudios entre $12 \%$ en los homocigotos y en $33 \%$ en los pacientes intolerantes a las estatinas. En ninguno de los estudios se observó aumento de la bilirrubina o alteración en la función de síntesis del hígado. El aumento en las transaminasas se ha correlacionado con la reducción en los niveles de ApoB, y ha sido motivo de discontinuación del fármaco en $<4 \%$ de los casos con $\mathrm{HFH}^{24}$. Por otra parte, mipomersen se ha asociado con un aumento variable y modesto del contenido graso en el hígado en algunos estudios, que se ha asociado con el mecanismo de acción del fármaco, variación en las transaminasas y tiempo de exposición al fármaco $^{24-28}$. Los estudios con resonancia magnética nuclear han demostrado la reversibilidad de la esteatosis hepática a largo plazo, tanto en diabéticos como en no diabéticos, sugiriendo cierto grado de adaptación del hígado ${ }^{27}$.

\section{Anticuerpos Monoclonales anti-Proproteína convertasa subtilisinakexina tipo 9 (PCSK9)}

Los RLDL son la principal vía de captación de las partículas de LDL desde la circulación. El C-LDL es captado por el receptor para luego ser internalizado y degradado en los lisosomas. Posteriormente, los RLDL son reciclados hacia la superficie del hepatocito.

La PCSK9 es una serina proteasa que tiene un rol clave en la degradación de los RLDL hepáticos, y por tanto, contribuye a regular los niveles plasmáticos de C-LDL. Esta proteína favorece la degradación intracelular de los RLDL, disminuyendo su reciclaje hacia la membrana plasmática del hepatocito ${ }^{29}$, lo que ocasiona una reducción del número de RLDL y por tanto, un aumento en los niveles de C-LDL. En el año 2003 se describieron algunas mutaciones en el gen PCSK9 que producen un aumento en la función de esta proteína y se asocian a hipercolesterolemia severa ${ }^{3}$. Posteriormente, se han descrito mutaciones en este gen que ocasionan una pérdida de función de la proteína, lo que se asocia a bajas concentraciones plasmáticas de C-LDL y a un menor riesgo coronario ${ }^{30,31}$.
Estos datos contribuyeron a la hipótesis de que la reducción o inhibición de PCSK9 podría ser un excelente objetivo terapéutico para reducir los niveles de C-LDL. Actualmente, se están desarrollando distintas estrategias para inhibir el PCSK9, siendo el bloqueo de esta proteína mediante anticuerpos monoclonales los que están en etapas más avanzadas de investigación ${ }^{32}$.

Los anticuerpos monoclonales que inhiben PCSK9, han sido desarrollados por varias empresas farmacéuticas y a partir del año 2012 se han publicado los resultados de estudios de fase I y II con AMG 145 de Amgen y SAR 236553/REGN 727 de Sanofi (Tabla 2).

La eficacia, seguridad y tolerabilidad de los anticuerpos monoclonales ha sido evaluada en estudios fase I en adultos sanos y en pacientes con hipercolesterolemia tratados con estatinas ${ }^{33,34}$. La reducción en C-LDL depende de la dosis, vía de administración e intervalo de frecuencia de dosis. Así, en sujetos sanos la administración endovenosa o sc de una dosis de AMG 145 produjo una reducción en C-LDL de hasta $64 \%$ similar a la conseguida con REGN727/SAR236553 vía endovenosa. En pacientes con hipercolesterolemia en tratamiento con estatinas, la reducción máxima en C-LDL conseguida fue de $75 \%$ con dosis repetidas de AMG145 vía sc cada 2 semanas y de $65 \%$ con REGN747 cada 4 semanas siendo algo menor en los pacientes con $\mathrm{HFH}^{33,34}$.

Estos resultados han sido confirmados por los estudios en fase II realizados en pacientes con $\mathrm{HFH}^{35,36}$, hipercolesterolemia con o sin tratamiento con estatinas ${ }^{37-40} \mathrm{y}$ en pacientes intolerantes a las estatinas ${ }^{41}$. Con AMG 145, la reducción en C-LDL máxima es de $66,1 \%$ y con REGN747 es de $72 \%$, dependiendo en ambos casos de la dosis, tratamiento concomitante e intervalo de administración, siendo superior cuando se administra cada 2 semanas. En el estudio de pacientes intolerantes a estatinas la reducción en C-LDL alcanzó 63\% cuando se administró la dosis máxima de AMG145 cada 4 semanas junto con ezetimiba ${ }^{41}$.

Adicionalmente, se ha observado una reducción significativa en los niveles de Lp(a) entre 20\% a $26 \%$, sin estar aun muy claros los mecanismos por los cuales se produce.

Actualmente, se están llevando a cabo estudios en fase III en pacientes con HFH y HFHo con ambas moléculas así como estudios con objetivos cardiovasculares. 


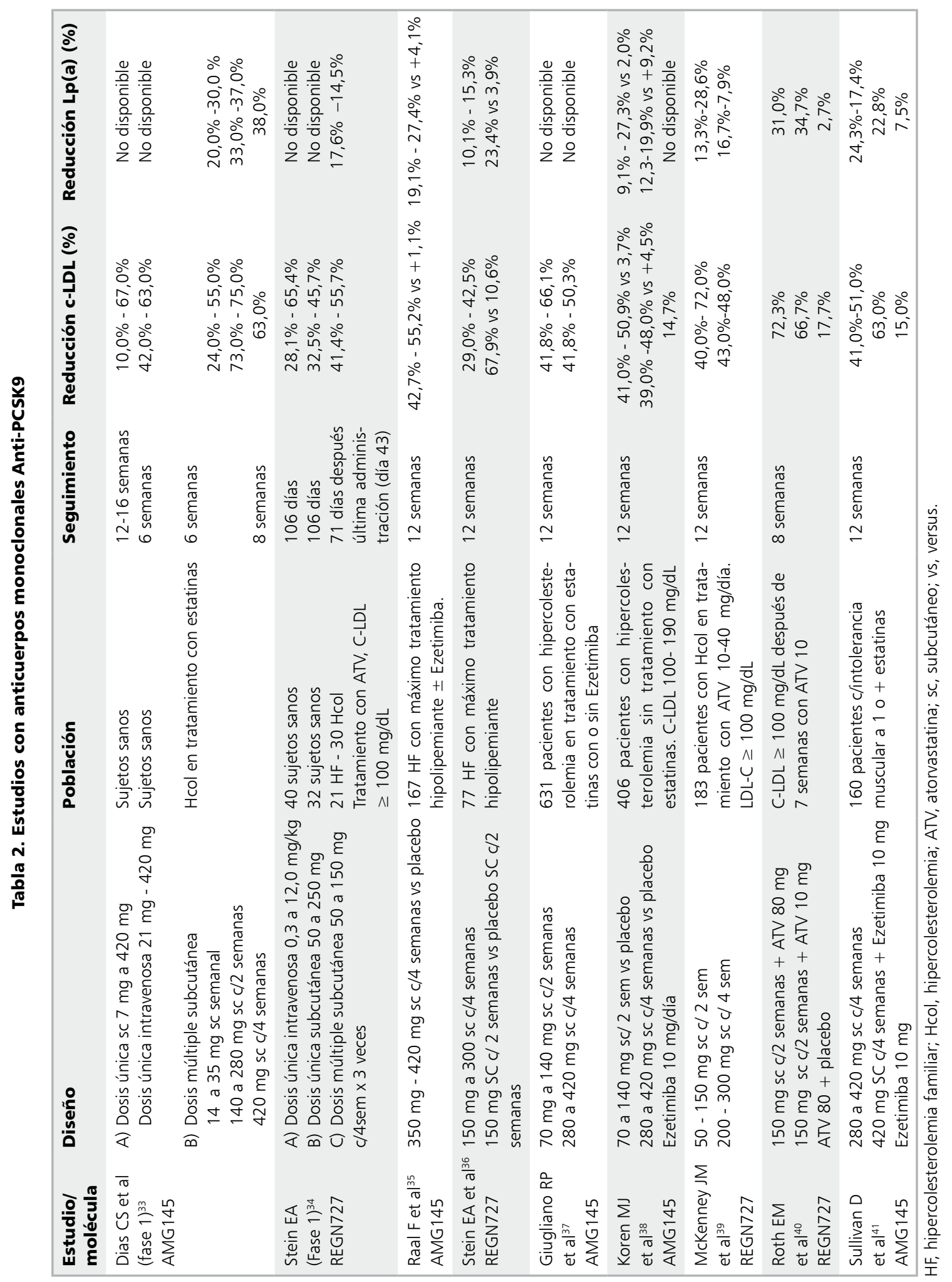


Los distintos estudios en fase I/II han mostrado que ambas moléculas son bien toleradas y no se han producido efectos adversos serios. No se deberían esperar interacciones con estatinas u otros fármacos ya que los anti-PCSK9 no se metabolizan por el Citocromo P450. En el estudio con AMG 145 en intolerantes a estatinas, se produjo $15 \%$ de mialgias aunque no se relacionó con la dosis del fármaco ${ }^{41}$. Se han reportado hasta la fecha casos esporádicos de prurito, aumento de CPK y vasculitis.

\section{Conclusiones}

Los nuevos tratamientos reductores de C-LDL que tienen mecanismos de acción novedosos y diferentes a los previamente conocidos, han surgido como terapia coadyuvante al tratamiento convencional en la hipercolesterolemia familiar homocigota o severa, e incluso en aquellos casos de alto riesgo cardiovascular con intolerancia al uso de estatinas. Los estudios a corto plazo han evidenciado su gran efecto hipolipemiante y un aceptable perfil de seguridad; sin embargo, se requieren estudios adicionales para establecer su beneficio y seguridad a largo plazo y principalmente su impacto en reducción de eventos cardiovasculares.

\section{Referencias}

1. Goldstein JL, Hobbs HH, Brown MS. Familial hypercholesterolemia. In: The metabolic and molecular basis of inherited disease. Editores: C. R. Scriver, A.L. Beaudet, W.S. Sly, and D. Valle. McGraw-Hill, New York. Vol. II, Chapter: 120. 2001; 2863-913.

2. Innerarity TL, Weisgraber KH, Arnolds KS, Mahley RW, Krauss RM, Vega GL, et al. Familial defective apolipoprotein B100: low density lipoproteins with abnormal receptor bindings. Proc Natl Acad Sci USA 1987; 84: 6919-23.

3. Abifadel M, Vartet M, Rabès JO, Allard D, Ouguerram $\mathrm{K}$, Devillers $\mathrm{M}$, et al. Mutations in PCSK9 cause autosomal dominant hypercholesterolemia. Nat Genet 2003; 34: 154-6.

4. Raal FJ, Santos RD. Homozygous familial hypercholesterolemia: current perspectives on diagnosis and treatment. Atherosclerosis 2012; 223: 262-8.

5. Dann EJ, Shamir R, Mashiach T, Shaoul R, Badian A,
Stravets T, et al. Early-onset plasmapheresis and LDLapheresis provide better control for pediatric homozygous familial hypercholesterolemia than HMG-CoA reductase inhhibitors and ameliorate atherosclerosis. Transfus Apher Sci 2013; 10.1016/j.transci.2013.05.001

6. Marais AD, Blom DJ, Firth J. Statins in homozygous familial hypercholesterolemia. Curr Atheroscler Rep 2002; 4: 19-25.

7. US Food and Drug Administration. Press Announcements. Disponible en: http://www.fda.gov/NewsEvents/ Newsroom/PressAnnouncements/ucm337195.htm [Consultado el 16 de septiembre de 2013].

8. The European Atherosclerosis Society. EAS 2013 Report. Disponible en: http://www.ema.europa.eu/docs/ en_GB/document_library/Summary_of_opinion_-_Initial_authorisation/human/002578/WC500143787.pdf [Consultado el 16 de septiembre de 2013].

9. Rizzo M. Lomitapide, a microsomal triglyceride transfer protein inhibitor for the treatment of hypercholesterolemia. Drugs 2010; 13: 103-11.

10. Hussain MM, Rava P, Walsh M, Rana M, Iqbal J. Multiple functions of microsomal triglycerides transfer protein. Nutr Metab (London) 2012; 9: 14. doi: 10.1186/1743-7075-9-14.

11. Berriot-Varoqueaux N, Aggerbeck LP, Samson-Bouma M. Microsomal triglycerides transfer protein and abetalipoproteinamia. Ann Endocrinol (Paris) 2000; 61: 125-9.

12. Cuchel M, Bloedon LT, Szapary PO, Kolansky DM, Wolfe ML, Sarkis A, et al. Inhibition of microsomal triglyceride transfer protein in familial hypercholesterolemia. N Engl J Med 2007; 356: 148-56.

13. Cuchel M, Meagher EA, du Toit Theron H, Blom DJ, Marais AD, Hegele RA, et al. Phase 3 HoFH Lomitapide Study investigators. Efficacy and safety of a microsomal triglyceride transfer protein inhibitor in patients with homozygous familial hypercholesterolaemia: a singlearm, open-label, phase 3 study. Lancet 2013; 381: 40-6.

14. Perry C. Lomitapide: A review of its use in adults with homozygous familial hypercholesterolemia. Am J Cardiovasc Drugs 2013; DOI 10.1007/s40256-013-0030-7.

15. Bennett CF, Swayze EE. RNA targeting therapeutics: molecular mechanisms of antisense oligonucleotides as a therapeutic platform. Annu Rev Pharmacol Toxicol 2010; 50: 259-93.

16. Davis RA. Cell and molecular biology of the assembly and secretion of apolipoprotein B-containing lipoproteins by the liver. Biochim Biophys Acta 1999; 1440: 1-31.

17. Visser ME, Witztum JL, Stroes ES, Kastelein JJ. Antisense oligonucleotides for the treatment of dyslipidaemia. Eur Heart J 2012; 33: 1451-8. 
18. Yu RZ, Kim TW, Hong A, Watanabe TA, Gaus HJ, Geary RS. Cross-species pharmacokinetic comparison from mouse to man of a second-generation antisense oligonucleotide, ISIS 301012, targeting human apolipoprotein B-100. Drug Metab Dispos 2007; 35 (3): 460-8.

19. Yu RZ, Geary RS, Flaim JD, Riley GC, Tribble DL, vanVliet AA, et al. Lack of pharmacokinetic interaction of mipomersen sodium (ISIS 301012), a 2'-Omethoxyethyl modified antisense oligonucleotide targeting apolipoprotein B-100 messenger RNA, with simvastatin and ezetimibe. Clin Pharmacokinet 2009; 48 (1): 39-50

20. Akdim F, Visser ME, Tribble DL, Baker BF, Stroes ES, Yu R, et al. Effect of mipomersen, an apolipoprotein B synthesis inhibitor, on lowdensity lipoprotein cholesterol in patients with familial hypercholesterolemia. Am J Cardiol 2010; 105: 1413-9.

21. Akdim F, Stroes E, Sijbrands E, Tribble D, Trip M, Jukema JW, et al. Efficacy and safety of Mipomersen, an Antisense Inhibitor os Apolipoprotein B, in hypercholesterolemia subjects receiving stable statin therapy. J Am Coll Cardiol 2010; 55: 1611-8.

22. Akdim F, Tribble DL, Flaim JD, Yu R, Su J, Geary RS, et al. Efficacy of apolipoprotein B synthesis inhibition in subjects with mild-to-moderate hyperlipidaemia. Eur Heart J 2011; 32: 2650-9.

23. Raal FJ, Santos RD, Blom DJ, Marais AD, Charng MJ, Cromwell WC, et al. Mipomersen, an apolipoprotein B synthesis inhibitor, for lowering of LDL cholesterol concentrations in patients with homozygous familial hypercholesterolaemia: a randomised, double-blind, placebo-controlled trial. Lancet 2010; 375 (9719): 9981006.

24. Stein EA, Dufour R, Gagne C, Gaudet D, East C, Donovan JM, et al. Apolipoprotein B synthesis inhibition with mipomersen in heterozygous familial hypercholesterolemia: results of a randomized, double-blind, placebo-controlled trial to assess efficacy and safety as add-on therapy in patients with coronary artery disease. Circulation 2012; 126 (19): 2283-92.

25. McGowan MP, Tardif JC, Ceska R, Burgess LJ, Soran $\mathrm{H}$, Gouni-Berthold I, et al. Randomized, placebocontrolled trial of mipomersen in patients with severe hypercholesterolemia receiving maximally tolerated lipid-lowering therapy. PLoS One 2012; 7 (11): e49006.

26. Visser ME, Wagener G, Baker BF, Geary RS, Donovan JM, Beuers UH, et al. Mipomersen, an apolipoprotein B synthesis inhibitor, lowers low-density lipoprotein cholesterol in high-risk statin-intolerant patients: a randomized, double-blind, placebo-controlled trial. Eur Heart J 2012; 33: 1142-9.
27. Thomas GS, Cromwell WC, Ali S, Chin W, Flaim JD, Davidson M. Mipomersen, an Apolipoprotein B Synthesis Inhibitor, Reduces Atherogenic Lipoproteins in Patients with Severe Hypercholesterolemia at High Cardiovascular Risk: A Randomized, Double-Blind, Placebo-Controlled Trial. J Am Coll Cardiol 2013 doi: 10.1016/j.jacc.2013.07.081.

28. Visser M, Akdim F, Tribble D, Nederveen A, Jesse Kwoh $\mathrm{T}$, Kastelein J, et al. Effect of apolipoproteína-B síntesis inhibition on liver triglyceride content in patients with familial hipercolesterolemia. J Lipid Res 2010; 51: 1057-62.

29. Surdo L, Bottomley MJ, Calzetta A, Settembre EC, Cirillo A, Pandit S, et al. Mechanistic implications forLDL receptor degradation from PCSK9/LDLR structure at neutral pH. EMBO Rep 2011; 12: 1300-5.

30. Cohen J, Pertsemlidis A, Kotowski IK, Graham R, Garcia CK, Hobbs HH. Low LDL cholesterol in individuals of African descent resulting from frequent nonsense mutations in PCSK9. Nat Genet 2005; 37: 161-5.

31. Cohen JC, Boerwinkle E, Mosley TH, Hobbs HH. Sequence variations in PCSK9, low LDL, and protection against coronary heart disease. N Engl J Med 2006; 354: 1264-73.

32. Marais D, Blom DJ, Petrides F, Gouëffic Y, Lambert G. Proprotein convertase subtilisin/kexin type 9 inhibition. Curr Opin Lipidol 2012; 23: 511-7.

33. Dias CS, Shaywitz AJ, Wasserman SM, Smith BP, Gao B, Stolman D, et al. Effects of AMG 145 on lowdensity lipoproteína cholesterol levels: results from 2 randomised,double-blind, placebo-controlled, ascending-dose phase 1 studies in healthy volunteers and hipercolesterolemic subjects on statins. J Am Coll Cardiol 2012; 60: 1888-98.

34. Stein EA, Mellis S, Yancopoulos GD, Stahl N, Logan $\mathrm{D}$, Smith WB, et al. Effect of a monoclonal antibody to PCSK9 on LDL cholesterol. N Engl J Med 2012; 366: 1108-18.

35. Raal F, Scott R, Somaratne R, Bridges I, Li G, Wasserman SM, et al. Low-density lipoproteína cholesterollowering effects of AMG 145, a monoclonal antibody to proprotein convertase subtilisin/kexin type 9 in patients with heterozygous familial hipercolesterolemia: the reduction of LDL-C with PCSK9 inhibition in heterozygous familial hipercolesterolemia disorder (RUTHEFORD) randomized trial. Circulation 2012; 126: 2408-17.

36. Stein EA, Gipe D, Bergeron J, Gaudet D, Weiss R, Dufour R, et al. Effect of a monoclonal antibody to PCSK9, REGN727/SAR236533, to reduce low-density lipoprotein cholesterol in patients with heterozygous familial hipercolesterolemia on stable statin dose with 
or without ezetimibe therapy: a phase 2 randomised controlled trial. Lancet 2012; 380: 29-36.

37. Giugliano RP, Desai NR, Kohli P,Rogers W, Somaratne R, Huang F, et al. Efficacy, safety, and tolerability of a monoclonal antibody to proprotein convertase subtili$\sin /$ kexin type 9 in combination with a statin in patients with hypercholesterolaemia (LAPLACE-TIMI 57): a randomised, placebo-controlled, dose-ranging, phase 2 study. Lancet 2012; 380: 2007-17.

38. Koren MJ, Scott R, Kim JB, Knusel B, Liu T, Bolognese $\mathrm{M}$, et al. Efficacy, safety, and tolerability of a monoclonal antibody to proprotein convertase subtilisin/kexin type 9 as monotherapy in patients with hypercholesterolaemia (MENDEL): a randomised, placebo-controlled, phase 2 study. Lancet 2012; 380: 1995-2006.
39. McKennay JM, Koren MJ, Kereiakes DJ, Hanotin C, Ferrand AC, Stein EA. Safety and efficacy of a monoclonal antibody to proprotein convertase subtilisin/kexin type 9, SAR 236553/REGN727, in patients with primary hipercolesterolemia receiving ongoing stable atorvastatin therapy. J Am Coll Cardial 2012; 59: 2344-53.

40. Roth EM, McKenney JM, Hanotin C, Asset G, Stein EA. Atorvastatin with or without an antibody to PCSK9 in primary hipercolesterolemia. N Engl J Med 2012; 367: 1891-900.

41. Sullivan D, Olsson AG, Scott R, Kim JB, Xue A, Gebski $\mathrm{V}$, et al. Effect of a monoclonal antibody to PCSK9 on low-density lipoproteína cholesterol levels in statin-intolerant patientes: the GAUSS randomized trial. JAMA 2012; 308: 2497-506. 